Full Length Article

\title{
Influence of weight gain on the modulation of wound healing following tooth extraction
}

\author{
Cristiane Furuse $^{\mathrm{a}}$, Aline F. Almeida ${ }^{\mathrm{b}}$, Sidnei F. Costa ${ }^{\mathrm{a}}$, Ana C. Ervolino-Silva ${ }^{\mathrm{a}}$, Roberta Okamoto ${ }^{\mathrm{c}}$, \\ Doris H. Sumidac ${ }^{c}$, Mariza A. Matsumoto ${ }^{c}$, Fábio R.M. Leite ${ }^{\mathrm{d}, *}$ \\ ${ }^{a}$ Department of Pathology and Clinical Propedeutics, Araçatuba Dental School, UNESP - São Paulo State University, Araçatuba, São Paulo, Brazil \\ ${ }^{\mathrm{b}}$ School of Dentistry, Federal University of Pelotas, Pelotas, RS, Brazil \\ ${ }^{\mathrm{c}}$ Department of Basic Sciences, Araçatuba Dental School, UNESP - São Paulo State University, Araçatuba, São Paulo, Brazil \\ d Section of Periodontology, Department of Dentistry and Oral Health, Aarhus University, Aarhus, Denmark
}

\section{A R T I C L E I N F O}

\section{Keywords:}

Tooth extraction

Immunohistochemistry

Repair

Obesity

\begin{abstract}
A B S T R A C T
Introduction: Obesity is characterized by extreme body fat accumulation related to lean body mass. The low-grade systemic inflammation induced by weight gain may influence wound healing. This study assessed the association between obesity and alveoli repair after tooth extraction.

Methods: Forty-two male Wistar rats were randomly divided into two groups: weight gain $(\mathrm{n}=21)$, animals fed with hyperlipidic cafeteria diet in order to gain weight; and control (non-obese; $n=21$ ) regularly fed rats. After twelve weeks, the upper right central incisor was extracted and animals were sacrificed after 7, 14 and 28 days. Slides were obtained for histological analysis. Bone formation and protein expression at the different periods were compared using Kruskal-Wallis test and Dunn post-test.

Results: Bone area was higher in the control group all over the experiment with more TRAP-positive cells and TRAP-positive labeling in the weight gain group. RANKL was homogeneously expressed along the experiment with no differences among the groups; conversely OPG levels reduced in the weight gain groups 14 and 28 days after tooth extraction. Osteocalcin labeling was higher in the control group after 7 days of extraction, with no differences at later time points. VEGF labeling was higher in the control group after 14 days of tooth extraction while the strongest immunolabeling in the weight gain group was observed 21 days post-extraction.

Conclusion: Weight gain induced a delay in bone repair after tooth extraction. The increase in the number of TRAP-positive cells observed in the extraction site seems to be mediated by the reduction in the expression of OPG rather than an overexpression of RANKL. In addition, the late expression of VEGF in the weight gain group might have delayed osteoblast migration and differentiation.
\end{abstract}

\section{Introduction}

Obesity is a chronic disease in which the body excessively accumulates fat [1], and a risk factor for many systemic diseases, such as type II diabetes, cardiovascular disease and cancer [2]. Obesity rates have risen considerably over the last decades becoming a global health problem [3].

The literature has demonstrated that obesity is associated with lowgrade chronic inflammation [4]. It has been hypothesized that the white adipose tissue is responsible for secreting constantly non-specific proinflammatory cytokines, such as interleukin (IL)-6, IL-1 beta and tumor necrosis factor-alpha (TNF-alpha), so as specific adipokines (leptin and adiponectin) [5]. Additionally, as a consequence of the expansion of the adipose tissue during weight gain, macrophages infiltrate the tissue, increasing the inflammatory load [5]. IL-1, IL-6 and IL- 8 can indirectly modulate the expression of the receptor activator of NF-kappa B ligand (RANKL) and osteoprotegerin (OPG) which may ultimately result in bone loss $[6,7]$.

Bone metabolism comprises the balance between resorption and formation, induced by osteoclasts and osteoblasts, respectively [8]. Bone turnover is mainly regulated by the RANK/RANKL/OPG axis [8]. Essentially, RANKL expressed on the osteoblast cell surface binds to RANK to stimulate osteoclast differentiation and maturation. On the other hand, osteoprotegerin (OPG) acts as a decoy receptor for RANKL, which in turn, prevents osteoclast differentiation and activation [9]. Thus, among the factors that may alter bone metabolism, chronic inflammation and increased inflammatory profile are responsible for inducing osteoclast activation, and therefore, bone resorption [10].

\footnotetext{
* Corresponding author.

E-mail address: fabio@dent.au.dk (F.R.M. Leite).
} 
Table 1

Biometric and plasma values (mean \pm standard deviation) after 28 days of tooth extraction.

\begin{tabular}{llll}
\hline & Control group & Weight gain group & P-value \\
\hline Weight $(\mathrm{g})$ & $509.4 \pm 40.7$ & $665.5 \pm 87.7$ & $0.01^{*}$ \\
Naso-anal measure $(\mathrm{cm})$ & $27.1 \pm 0.32$ & $27.4 \pm 0.9$ & 0.56 \\
Lee index & $294.1 \pm 4.7$ & $318.2 \pm 11.9$ & $0.01^{*}$ \\
PWAT (g) & $6.6 \pm 0.5$ & $26.8 \pm 6.6$ & $0.01^{*}$ \\
RWAT (g) & $6.1 \pm 0.6$ & $39.8 \pm 16.1$ & $0.01^{*}$ \\
Glycaemia (mg/dL) & $70.0 \pm 3.5$ & $93.8 \pm 11.9$ & $0.01^{*}$
\end{tabular}

PWAT - parametrial white adipose tissue, RWAT - retroperitoneal white adipose tissue.

The relationship between obesity and bone metabolism is still controversial within the literature. Initially, obesity was described as beneficial to bone tissue, since mechanical loading conferred by body weight would stimulate bone formation [11]. However, studies have also identified detrimental effects of obesity on bone metabolism [12, 13]. According to the literature, the chronic inflammatory frame combined with increased levels of proinflammatory cytokines induced by excessive body weight might nullify potential beneficial effects of obesity on bone turnover [8]. This detrimental impact of obesity on bone metabolism may be more perceived in body locations that do not experience the benefits of mechanical loading, such as the alveolar bone. Studies have demonstrated that obesity may induce alveolar bone loss in rats [14, 15], and in humans [16].

Even though studies have demonstrated the effects of excessive body weight on bone loss, there is a lack of information about the influence of obesity on the mechanisms involved in bone healing, more specifically in the alveolar bone. Given the existing gap in the relationship between obesity and bone repair, and the risen prevalence of obesity worldwide, it is of relevance to understand such relationship. Accordingly, this study aimed to investigate whether obesity would influence bone metabolism. Also, to explore mechanisms underlying this association.

\section{Methods}

\subsection{Animals}

This study was approved by the Animal Research Ethics Committee
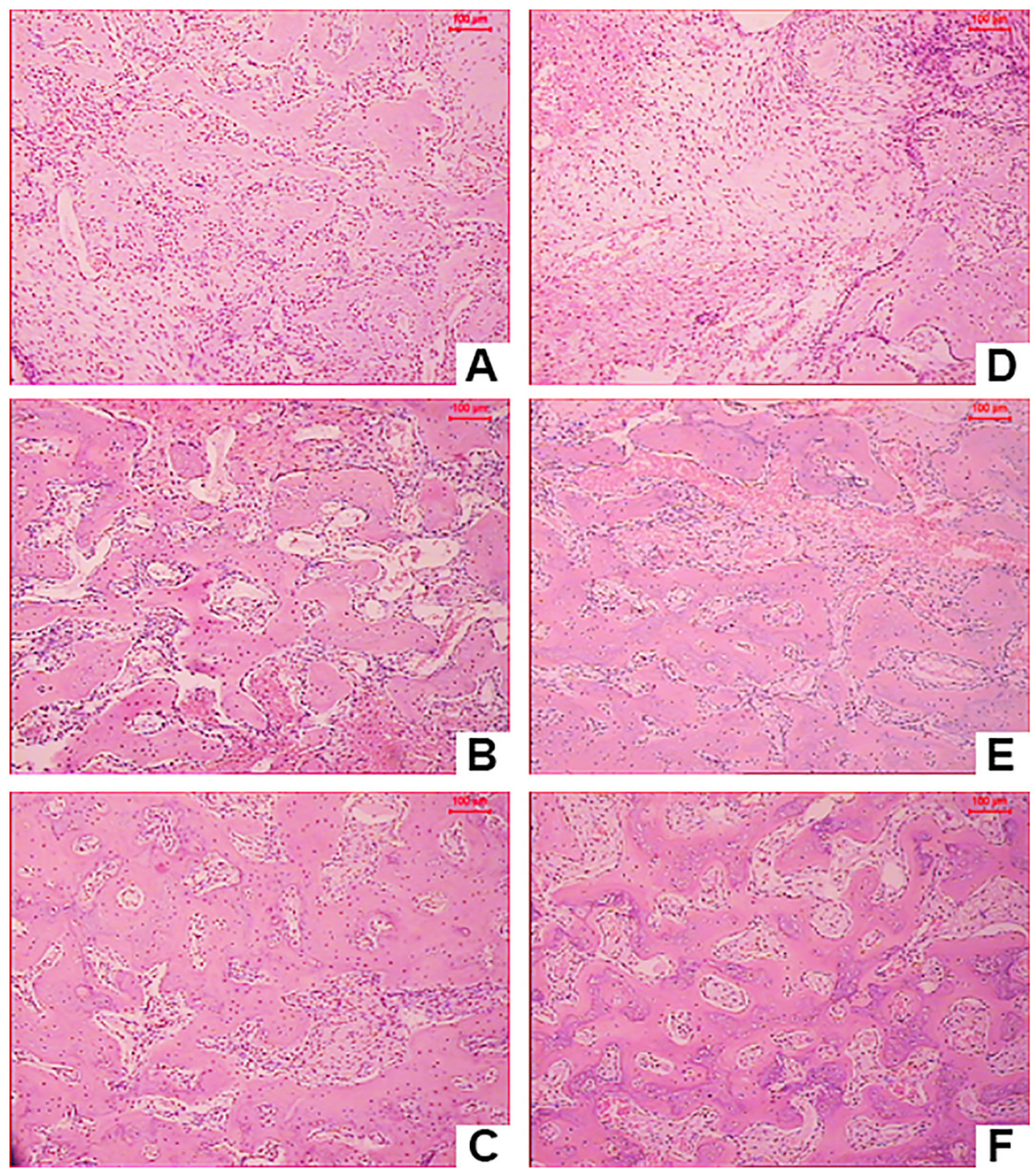

Fig. 1. Photomicrographs illustrating the chronology of alveolar bone repair at 7, 14 and 28 days post-extraction, from the control group (A, B, C) and weight gain group (D, E, F) (HE, original magnification $100 \times$ ). 
Table 2

Mean and standard deviation data regarding bone area formation and the number of TRAP positive cells.

\begin{tabular}{llll}
\hline & Control group & Weight gain group & P-value \\
\hline Bone area 7 days $\left(\mathrm{mm}^{2}\right)$ & $35.0 \pm 6.6$ & $15.8 \pm 5.5$ & $0.01^{*}$ \\
Bone area 14 days $\left(\mathrm{mm}^{2}\right)$ & $47.1 \pm 5.7$ & $31.8 \pm 8.0$ & $0.01^{*}$ \\
Bone area 28 days $\left(\mathrm{mm}^{2}\right)$ & $57.2 \pm 6.9$ & $40.4 \pm 16.4$ & $0.05^{*}$ \\
TRAP positive cells 7 days & $111.2 \pm 24.9$ & $120.6 \pm 53.8$ & 0.73 \\
TRAP positive cells 14 days & $216.4 \pm 54.8$ & $261.0 \pm 115.4$ & 0.46 \\
TRAP positive cells 28 days & $156.4 \pm 49.2$ & $237.4 \pm 70.6$ & $0.05^{*}$ \\
\hline
\end{tabular}

TRAP - tartrate-resistant acid phosphatase.

Table 3

Median and range data regarding immunohistochemistry labelling scores.

\begin{tabular}{llll}
\hline & Control group & Weight gain group & P-value \\
\hline OPG 7 days & $2[2-2]$ & $2[2-2]$ & 1.00 \\
OPG 14 days & $3[3-3]$ & $2[2-3]$ & $0.01^{*}$ \\
OPG 28 days & $3[3-3]$ & $1[1-2]$ & $0.01^{*}$ \\
RANKL 7 days & $3[3-3]$ & $3[2-3]$ & 0.13 \\
RANKL 14 days & $3[2-3]$ & $3[2-3]$ & 0.51 \\
RANKL 28 days & $3[2-3]$ & $3[2-3]$ & 1.00 \\
TRAP 7 days & $1[1-1]$ & $1[1-2]$ & 0.13 \\
TRAP 14 days & $2[1-2]$ & $2[2-3]$ & $0.05^{*}$ \\
TRAP 28 days & $2[1-2]$ & $3[3-3]$ & $0.01^{*}$ \\
OC 7 days & $3[3-3]$ & $2[2-3]$ & $0.01^{*}$ \\
OC 14 days & $3[2-3]$ & $2[2-3]$ & 0.22 \\
OC 28 days & $3[2-3]$ & $2[2-3]$ & 0.22 \\
VEGF 7 days & $1[1-2]$ & $1[1-2]$ & 0.51 \\
VEGF 14 days & $2[2-3]$ & $2[1-2]$ & $0.05^{*}$ \\
VEGF 28 days & $1[1-2]$ & $2[2-3]$ & $0.01^{*}$ \\
\hline
\end{tabular}

OPG - osteoprotegerin, RANKL - receptor activator of NF-kappa B ligand, TRAP - tartrate-resistant acid phosphatase, OC - osteocalcin, VEGF - vascular endothelial growth factor.

of the Araçatuba Dental School, UNESP, Brazil (Protocol number 2014/ 00293). Experiment was conducted under Good Laboratory Practice (GLP) conditions and in accordance with the guidelines of the USA National Research Council and the Canadian Council on Animal Care (CCAC).

Forty-two male Wistar rats with approximately 60 days of age and weighting approximately $250 \mathrm{~g}$ were used in this study. The sample size calculation considered the variability of measurements of alveolar bone repair, and acceptance as significant differences between experimental groups of $2 \mathrm{~mm}$, with alpha and beta errors of 0.05 and 0.2 , respectively [17]. The number of animals in each group was estimated in six. Based on attrition rates observed in our previous studies, seven animals were included in each group.

Animals were allocated into two groups according to body weight. A stratified randomization strategy comprising tertiles of body weight was used in order to minimize possible group impairment at baseline. The control group received a standardized rat chow (Nuvilab, Curitiba, PR, Brazil), which consisted of $58 \%$ carbohydrates, $20 \%$ protein, $5 \%$ fat and $5 \%$ of other constituents (vitamins, minerals and preservatives). The weight gain group received a high fat and hypercaloric diet, also known as CAF diet. This hypercaloric diet, also known as cafeteria (CAF) diet or Western diet, consisted of commercial rat chow (Hiperlipídica M 42\%, Rhoster, Araçoiaba da Serra, SP, Brazil) plus sausage and sweet biscuit in the proportion of 2:2:1. Diet consisted of approximately $50 \%$ carbohydrates, $20 \%$ fat, $20 \%$ proteins and $5 \%$ of other constituents, adapted from a hyperlipidic diet [14, 15]. All foods were available ad libitum for both obesity and control groups.
The animals received the standard or hypercaloric diet for 12 weeks. Weight and length according to the naso-anal measure were obtained every two weeks, and allowed the calculation of the Lee index. The Lee index consists of the ratio of the cube root of the weight in grams by the naso-anal length in centimeters multiplied by 1000 . Animals with Lee index above 300 were considered obese [18]. Obesity was confirmed by weighting the parametrial white adipose tissue (PWAT) and retroperitoneal white adipose tissue (RWAT) after euthanasia. A comprehensive study flowchart is presented in Supplemental Fig. 1.

\subsection{Glycaemia monitoring}

Blood glucose levels were measured by an automatic monitoring system at preoperative and postoperative time periods (7, 14 and 28 days) (Accu-Check ${ }^{\circledast}$ Performa; Roche-Diagnostics Corporation, Indianapolis, IN, USA).

\subsection{Surgery}

After 12 weeks of diet exposure, animals were anesthetized by intramuscular administration of a solution of ketamine chloride $(50 \mathrm{mg}$ / $\mathrm{kg}$, Vetbrands, Jacareí, SP, Brazil) and xylazine chloride $(10 \mathrm{mg} / \mathrm{kg}$, Coopers Brasil LTDA, São Paulo, SP, Brazil). Anterior maxilla antisepsis was performed using iodized polyvinylpyrrolidone and extraction of the upper right incisor was done using specially adapted tools, as previously described [19]. The margins of the wound were sutured with nylon wires (Vicryl 5-0, Ethycon, São Paulo, SP, Brazil).

The alveolar bone repair in rat incisors area has been studied for the past five decades [19]. Because the chronology and the cellular responses of the bone repair are well characterized, this model became interesting to evaluate systemic factors that may interfere in the process. In addition, in comparison to the molar area, the incisor region is of easier access to identify postsurgical complications, and to avoid damages to nearby teeth during the extraction procedure.

\subsection{Sample collection}

Animals were euthanized after 7, 14 and 28 days of tooth extraction in chamber saturated with halothane vapor. White adipose tissue from retroperitoneal (RWAT) and parametrial (PWAT) regions were collected and weighed to assure weight gain due to fat accumulation. Next, the upper maxilla was split in two by means of median sagittal incision following the intermaxillary suture. Alveolar bone samples were obtained through tangential cuts to the molar distal faces using surgical scissors. Samples were immersed in $10 \%$ buffered formalin solution ( $\mathrm{pH}$ 7.0) for $48 \mathrm{~h}$ for histological processing.

\subsection{Histomorphometric analysis}

Samples were decalcified in buffered (pH 8) 17\% EDTA (Sigma Chemical Co; St Louis, MO, USA) and embedded in paraffin. Semiserial longitudinal $6 \mu \mathrm{m}$-thick sections were stained with hematoxylin and eosin (H\&E). The histomorphometric analysis of the bone area in the middle thirds of the rat alveolus was performed in three sections from each animal. Standardization of analysis was determined by splitting the length of the alveolus into thirds. Alveolus walls were recognized by their regular contour in the early time points and by reversal lines when trabecular fill-in was present [20]. Upper and lower alveolar walls were determined by drawing a virtual line connecting the buccal and lingual alveolar crests to the most superior and occlusal points of the alveolar base, respectively [20]. Subsequently, the lines were equally split in three and the corresponding points from upper and lower lines connected [21] (Supplemental Fig. 2). Two digital photomicrographs were 

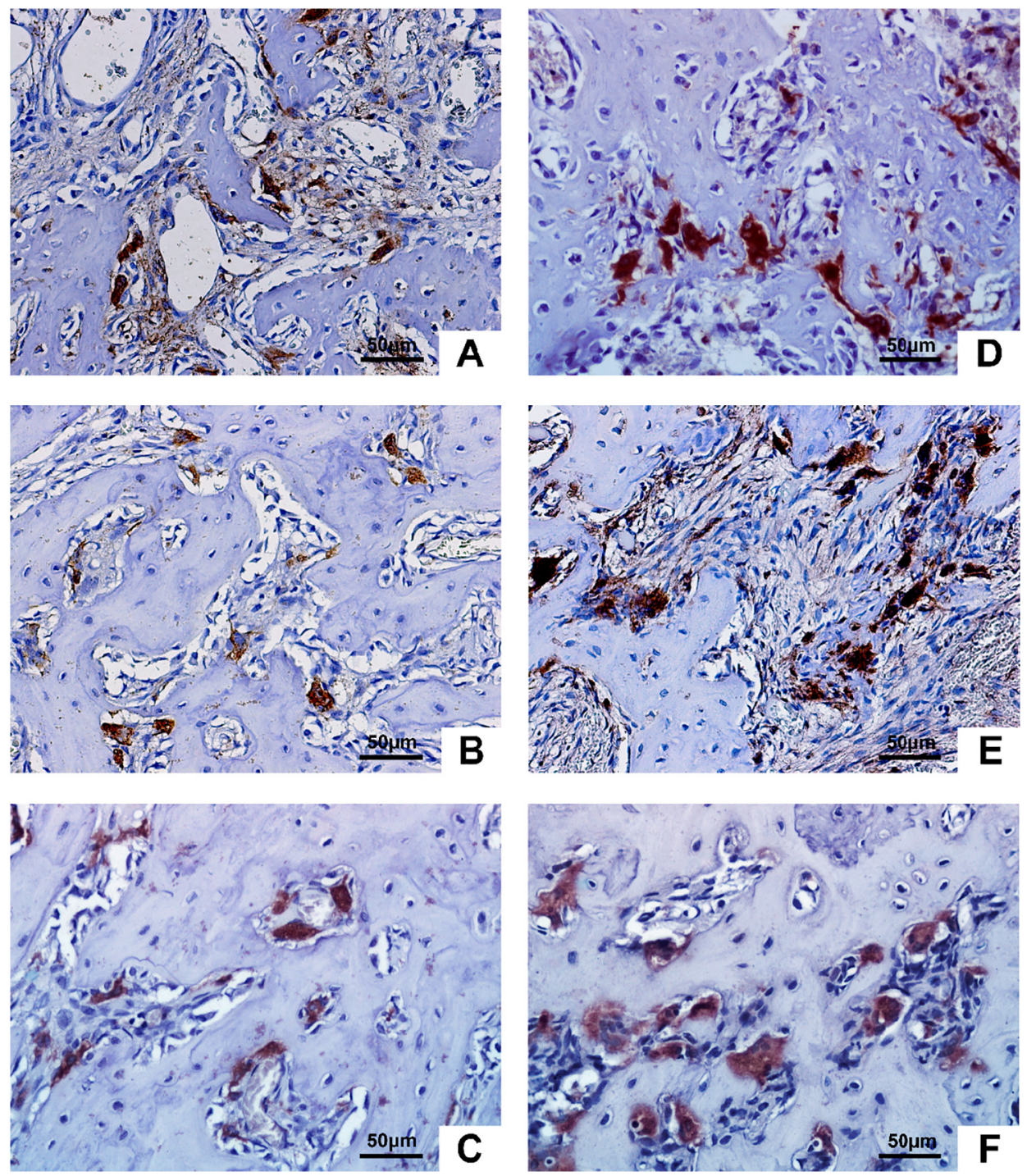

Fig. 2. Illustrative images of tartrate-resistant acid phosphatase (TRAP) immunolabeling after 7, 14 and 28 of tooth extraction in control group (A, B, C) and weight gain group (D, E, F) (original magnification $\times 400$ ).

captured at the center of the delimited area with the field of view touching the anterior and posterior delimitation lines. The analysis was performed with an optical microscope Leica Aristoplan Microsystems (Leitz, Benshein, Germany) with a magnification objective of $10 \times$, coupled to an image-capturing camera (Leica DFC 300FX, Leica Microsystems, Heerbrugg, Switzerland). Digitized images were analyzed in a specific software (Leica Camera Software Box, Leica Imaging Manager 50). An examiner under blinded conditions performed the analysis.

\subsection{Immunohistochemical analysis}

The sections next to the H\&E-stained section were used for immunohistochemical staining to verify the expression of OPG, RANKL, osteocalcin (OC), tartrate-resistant acid phosphatase (TRAP) and vascular endothelial growth factor (VEGF) in the tissues during the healing process. After deparaffinization, the slides were washed in phosphatebuffered saline, blocked with $0.03 \%$ hydrogen peroxide (Merck, Darmstadt, Germany) and submitted to antigen recovery. Nonfat milk was used to block endogen biotin. Goat polyclonal anti-OPG, antiRANKL, anti-osteocalcin, anti-TRAP and anti-VEGF antibodies (Santa Cruz Biotechnology, Santa Cruz, CA, USA) were used. The secondary antibody was biotinylated anti-goat antibody (Santa Cruz Biotechnology, Santa Cruz, CA, USA).

Reactions were amplified using avidin-biotin immunoperoxidase (Dako Corp., Carpinteria, CA, USA) and developed by diaminobenzidine (Dako Corp.). Sections were counterstained with hematoxylin (Merck), dehydrated, and coverslips were mounted using Permount (Fisher Scientific Co., NJ, USA). Analysis was performed to identify the labelling characteristics of each protein.

Three slides of each specimen were selected for analysis of OPG, RANKL, osteocalcin, TRAP and VEGF expression. The area corresponding to the extraction socket was examined. The immunostaining intensity was categorized by a blinded calibrated examiner according to a scale from 1 to 4 attributed to absent/negligible, weak, moderate, and strong staining, respectively [22]. 

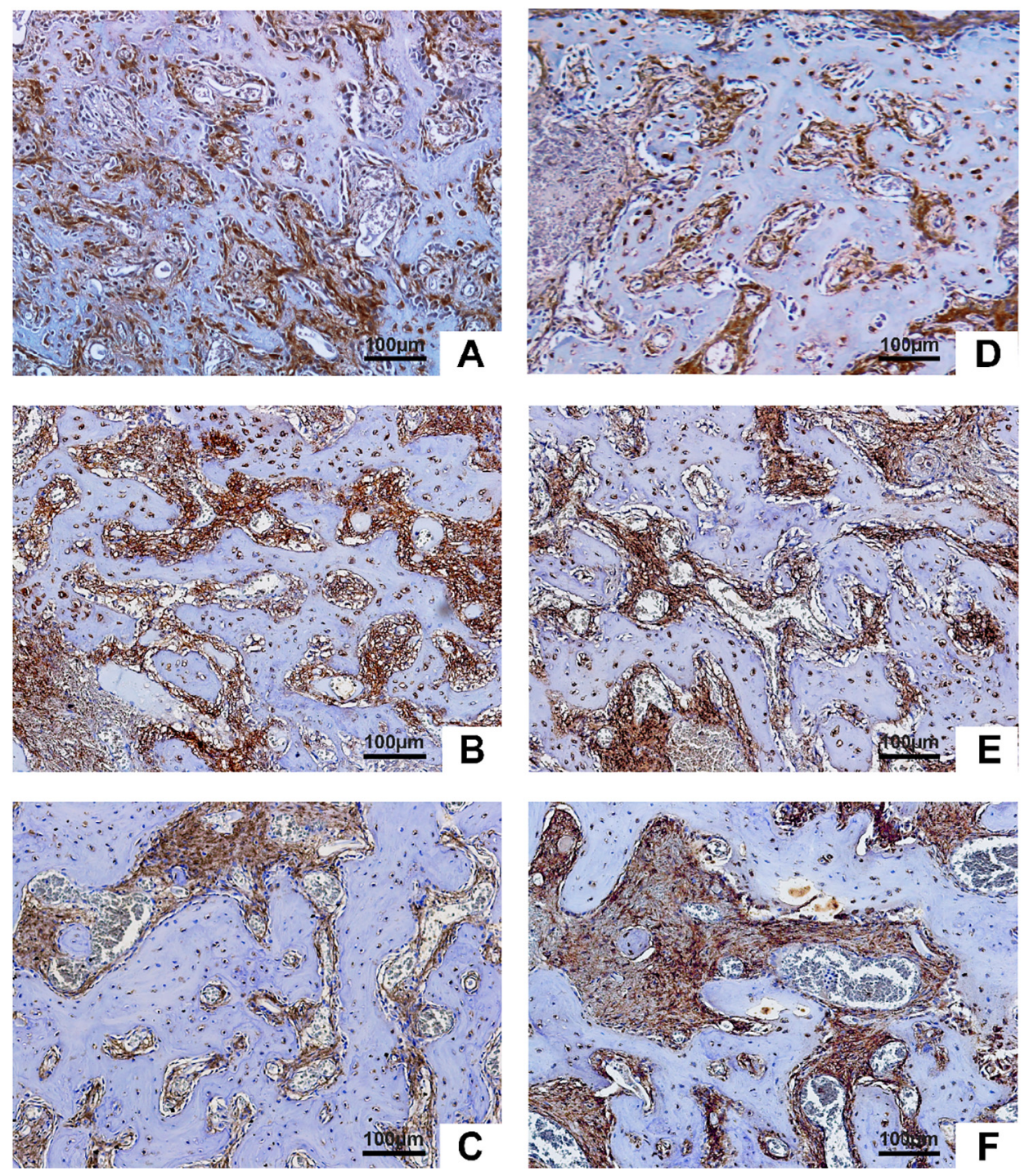

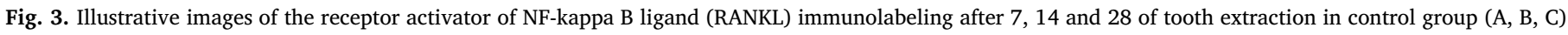
and weight gain group (D, E, F) (original magnification $\times 200$ ).

\subsection{Statistical analysis}

Normality was tested by Shapiro-Wilk test. Mean body weight and Lee Index were calculated for control and weight gain groups and compared by independent samples $t$-test. Bone formation and protein expression at the different periods were compared using the nonparametric Kruskal-Wallis test and Dunn post-test, employing StataSE 14.1 software (StataSoft, College Station, TX, US). The significant level was set at $5 \%$.

\section{Results}

At baseline, before the animals were allocated to different feeding groups, no statistical differences were observed in weight and length between the control and the weight gain groups (data not shown). Animals in the weight gain group increased weight by the accumulation of abdominal adipose tissue. Glycaemia levels among obese rats were higher than in control animals (Table 1).

After 7 days of tooth extraction, both groups showed an initial repair of the extraction socket. The onset of alveolar bone repair was characterized by the presence, mainly in the periphery of the alveolus, of some trabeculae of osteoid material and immature bone tissue, with numerous osteocytes and irregular basophilic apposition lines, permeated by densely cellularized connective tissue still presenting hemorrhagic areas.

At 14 days, the bone tissue trabeculae started to show anastomosis and filled most of the alveolus. The connective tissue was still well densely cellularized and some smaller hemorrhagic areas could still be observed. After 28 days of tooth extraction, the anastomosed bone trabeculae were thicker, although still immature, and there was less osteoid material. The connective tissue was more scarce and mature, showing a more fibrous characteristic with less cellularity.

Small differences were observed between the specimens of the control group and the weight gain group, with the latter always showing a more delayed repair. The Fig. 1 illustrates the overall evolution of tissue repair.

Bone area was higher in the control group all over the experiment with more TRAP-positive cells and TRAP-positive labeling in the weight 

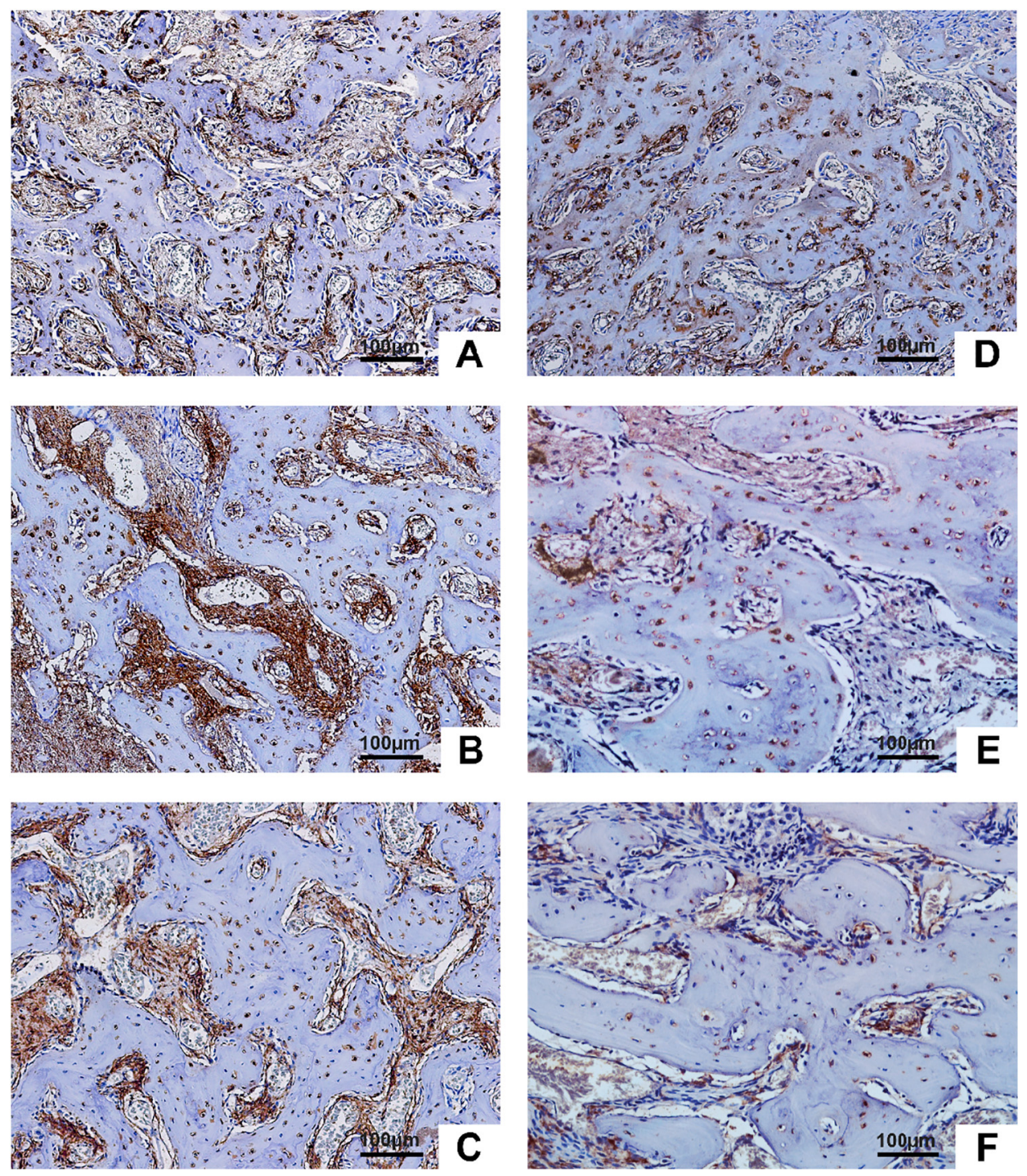

Fig. 4. Illustrative images of osteoprotegerin (OPG) immunolabeling after 7, 14 and 28 of tooth extraction in control group (A, B, C) and weight gain group (D, E, F) (original magnification $\times 200$ ).

gain group (Tables 2 and 3 and Fig. 2). RANKL was homogeneously expressed along the experiment with no differences among the groups (Fig. 3), conversely OPG levels reduced in the weight gain groups 14 and 28 days after tooth extraction (Table 3 and Fig. 4). Osteocalcin labeling was higher in the control than the weight gain group after 7 days of tooth extraction, with no differences at later time points (Table 3 and Fig. 5). VEGF expression was higher in the control group after 14 days of tooth extraction while the strongest immunolabeling in the weight gain group was observed 21 days post-extraction (Table 3 and Fig. 6).

\section{Discussion}

Even though obesity was thought to be a protecting factor for the skeleton due to its association with increased bone mineral density [23], it has been recently shown that obesity is associated with increased prevalence of bone fractures [24]. A recent prospective cohort study using data from 56,492 fractures of Medicare patients identified obesity as a risk factor for fracture nonunion [25]. The reduced bone turnover seems to be related with progressive insulin resistance induced by weight gain [26].

In our study, CAF diet-induced obesity was associated with a delay in bone repair after tooth extraction in Wistar rats. Obesity might affect bone metabolism by different mechanisms. Osteoblastogenesis is inversely related with adipogenesis since adipocytes and osteoblasts have a common mesenchymal stem cell origin [27]. Moreover, adipocyte proliferation constrains blood vessels compromising cellular nutrition, which may cause foci of necrosis in the adipose tissue [28]. Macrophages are recruited to necrosis sites releasing in the bloodstream proinflammatory cytokines release, e.g. IL- 6 and TNF- $\alpha$ [28, 29].

These proinflammatory cytokines stimulate osteoclast differentiation and activity through the modulation of the RANKL/RANK/OPG pathway [6, 7]. Increased RANKL:OPG ratios determine osteoclastogenesis and bone resorption through the nuclear factor $\mathrm{\kappa B}$ and protein kinase B-mediated signaling pathways [6, 7]; whereas reduced RANKL:OPG ratios favor bone formation. In our study, the RANKL:OPG ratio was increased in the weight gain group and as a result, bone repair was delayed. Our results corroborate with a previous study [30], which 

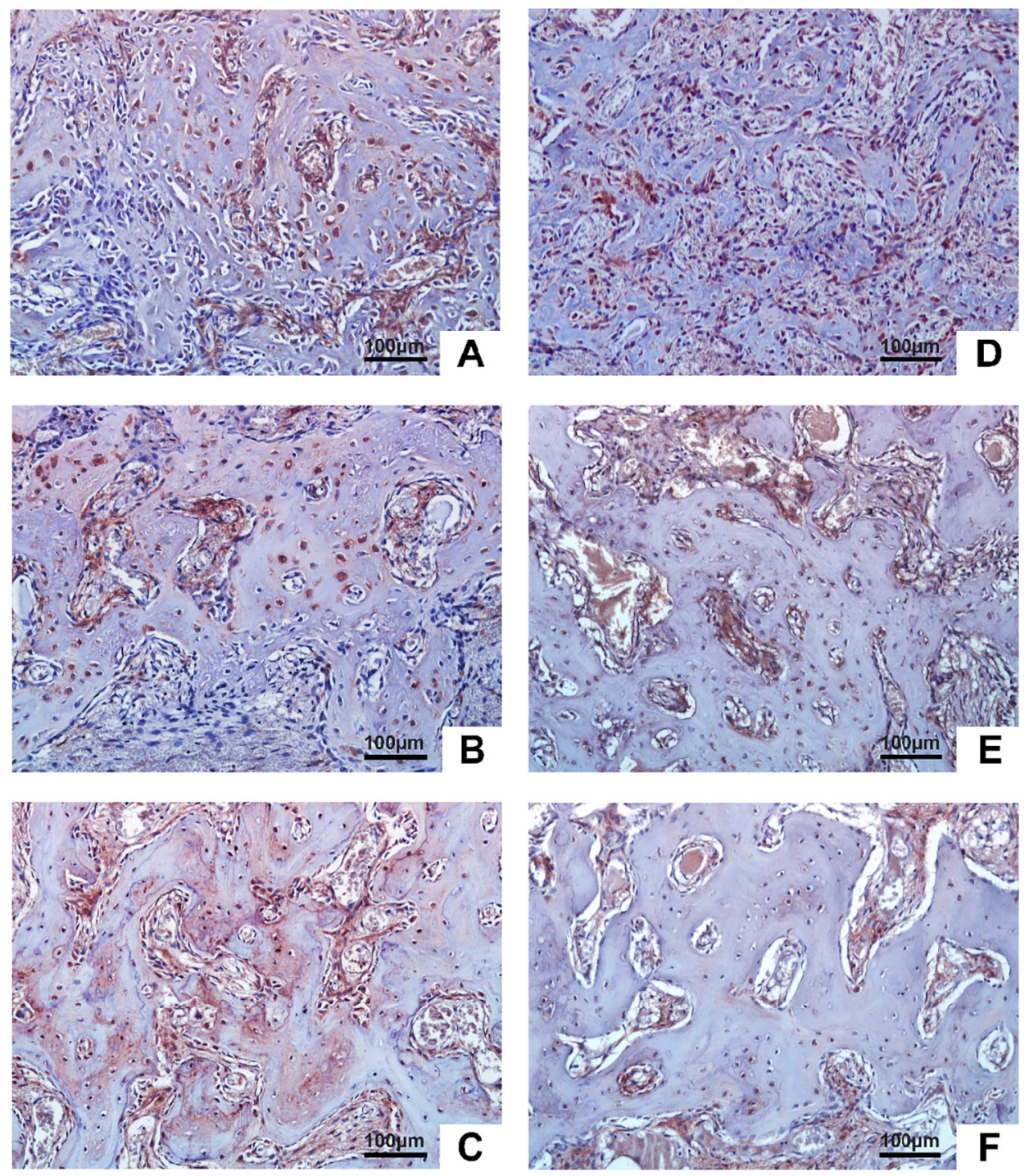

Fig. 5. Illustrative images of osteocalcin (OC) immunolabeling after 7, 14 and 28 of tooth extraction in control group (A, B, C) and weight gain group (D, E, F) (original magnification $\times 200$ ).

reported a decrease in OPG secretion in a model of obesity simulation, even though other studies [30-32] identified an increase in RANKL level. Regardless of the RANKL:OPG ratio component affected, results point out that the reduced number of trabecular bone formed in the weight gain group might be explained by the elevated number of TRAPpositive cells compared to the control group. TRAP has been used as a marker of bone turnover since it participates in the degradation of collagen intracellularly in osteoclasts $[33,34]$ and in the osteoclast adhesion and migration when secreted in the extracellular matrix [35]. Based on that, we decided to count TRAP-positive cells and to score the TRAP stained area. It is important to acknowledge the differences in the methodologies. Scoring the stained area is less prone to errors than isolating and counting TRAP positive cells. To avoid an overestimation of cell number, when in doubt in the number of cells due to proximity or overlap, only one was counted even though an underestimated result can be obtained. It is important to highlight also that doubts occurred more frequently in the weight gain than in the control group.

The delay in bone formation and maturation was also confirmed by osteocalcin deposition. Osteocalcin is synthesized by osteoblasts along bone formation and it is used as a biomarker for the bone remodeling process [36]. Control group presented OC precipitated all over the new bone matrix inside the extraction socket as expected. Conversely, in the weight gain group even though labeling scores were still high due to osteoblast labeling, the mineralized matrix was poorly impregnated. New theories suggest that OC is a bone-derived hormone related with the regulation of the metabolism [37]. OC may be used to control insulin secretion, $\beta$-cell proliferation, and triglyceride levels in obesity [37], which may explain OC reduced expression in bone matrix in our rats. Furthermore, osteoblast chemotaxis, differentiation, and matrix mineralization are stimulated by VEGF [38]. Given the late expression of VEGF in the weight gain group, a delayed socket repair would be expected among these animals.

Based on our results, obesity induced a delay in bone repair after tooth extraction. The increase in the number of TRAP-positive cells in the extraction site seems to be mediated by the reduction in the expression of OPG rather than an overexpression of RANKL. Furthermore, the delayed expression of VEGF might have affected osteoblasts chemotaxis and differentiation. Results must be explored in clinical trials to 

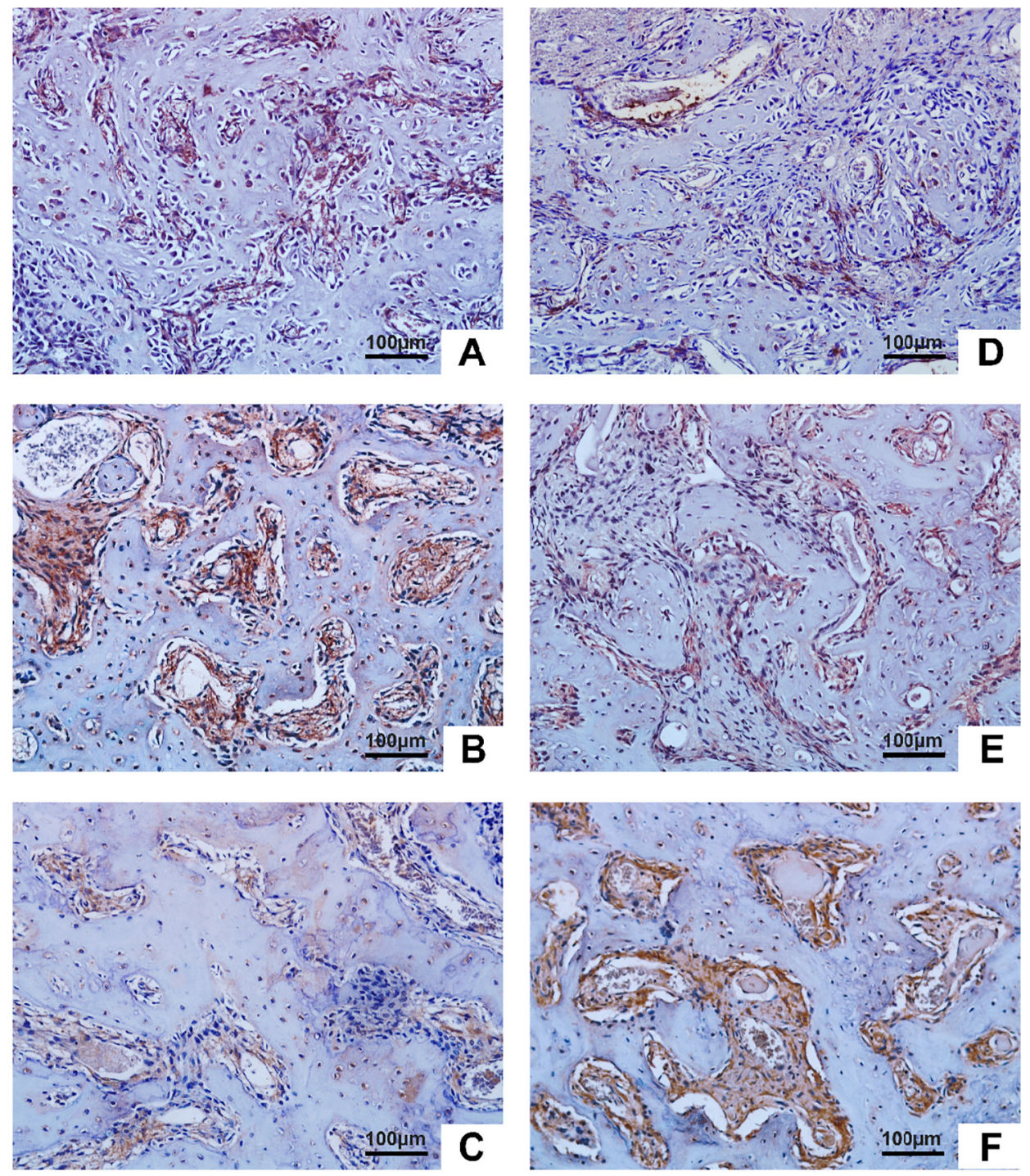

Fig. 6. Illustrative images of vascular endothelial growth factor (VEGF) immunolabeling after 7, 14 and 28 of tooth extraction in control group (A, B, C) and weight gain group (D, E, F) (original magnification $\times 200$ ).

confirm the delay in alveolar bone healing and to determine a possible impact of weight gain in dental treatments.

\section{Funding}

This work was supported by PROPE-UNESP and FUNDUNESP [grant 0345/001/14].

\section{Declarations of interest}

None.

\section{Authors' contributions}

FRML and CF conceived the project, designed the study and wrote the manuscript. AFA, SFC, ACES, RO performed experiments, analyzed data and edited the manuscript. DHS and MAA applied for grants, performed experiments, analyzed the data and edited the manuscript.

\section{Appendix A. Supplementary data}

Supplementary data to this article can be found online at https:// doi.org/10.1016/j.bone.2018.06.017.

\section{References}

[1] World Health Organization, Obesity: Preventing and Managing the Global Epidemic. Report of the World Health Organization Consultation of Obesity, WHO, Geneva, Switzerland, 2000.

[2] S.G. Chrysant, G.S. Chrysant, New insights into the true nature of the obesity paradox and the lower cardiovascular risk, J. Am. Soc. Hypertens. 7 (1) (2013) 85-94.

[3] D.W. Haslam, W.P. James, Obesity, Lancet 366 (9492) (2005) 1197-1209.

[4] P.G. Kopelman, Obesity as a medical problem, Nature 404 (6778) (2000) 635-643.

[5] G.S. Hotamisligil, Inflammation and metabolic disorders, Nature 444 (7121) (2006) $860-867$.

[6] F.R.M. Leite, S.G.d. Aquino, M.R. Guimarães, J.A. Cirelli, C.R. Junior, RANKL expression is differentially modulated by TLR2 and TLR4 signaling in fibroblasts and osteoblasts, Immunol. Innov. 2 (1) (2014).

[7] F.R.M. Leite, S.G. de Aquino, M.R. Guimarães, J.A. Cirelli, D.S. Zamboni, J.S. Silva, C.R. Junior, Relevance of the myeloid differentiation factor 88 (MyD88) on RANKL, OPG, and nod expressions induced by TLR and IL-1R signaling in bone marrow stromal cells, Inflammation (2014) 1-8.

[8] J.J. Cao, Effects of obesity on bone metabolism, J. Orthop. Surg. Res. 6 (2011) 30.

[9] D.L. Lacey, E. Timms, H.L. Tan, M.J. Kelley, C.R. Dunstan, T. Burgess, R. Elliott, A. Colombero, G. Elliott, S. Scully, H. Hsu, J. Sullivan, N. Hawkins, E. Davy, C. Capparelli, A. Eli, Y.X. Qian, S. Kaufman, I. Sarosi, V. Shalhoub, G. Senaldi, J. Guo, J. Delaney, W.J. Boyle, Osteoprotegerin ligand is a cytokine that regulates osteoclast differentiation and activation, Cell 93 (2) (1998) 165-176.

[10] D.V. Novack, S.L. Teitelbaum, The osteoclast: friend or foe? Annu. Rev. Pathol. 3 (2008) 457-484.

[11] D.T. Villareal, C.M. Apovian, R.F. Kushner, S. Klein, N. American Society for, T.O.S. Naaso, Obesity in older adults: technical review and position statement of the 
American Society for Nutrition and NAASO, The Obesity Society, Obes. Res. 13 (11) (2005) 1849-1863.

[12] Y.H. Hsu, S.A. Venners, H.A. Terwedow, Y. Feng, T. Niu, Z. Li, N. Laird, J.D. Brain, S.R. Cummings, M.L. Bouxsein, C.J. Rosen, X. Xu, Relation of body composition, fat mass, and serum lipids to osteoporotic fractures and bone mineral density in Chinese men and women, Am. J. Clin. Nutr. 83 (1) (2006) 146-154.

[13] N.K. Pollock, E.M. Laing, C.A. Baile, M.W. Hamrick, D.B. Hall, R.D. Lewis, Is adiposity advantageous for bone strength? A peripheral quantitative computed tomography study in late adolescent females, Am. J. Clin. Nutr. 86 (5) (2007) 1530-1538.

[14] J. Cavagni, T.P. Wagner, E.J. Gaio, R.O. Rego, I.L. Torres, C.K. Rosing, Obesity may increase the occurrence of spontaneous periodontal disease in Wistar rats, Arch. Oral Biol. 58 (8) (2013) 1034-1039.

[15] J. Cavagni, I.C. de Macedo, E.J. Gaio, A. Souza, R.S. de Molon, J.A. Cirelli, A.L. Hoefel, L.C. Kucharski, I.L. Torres, C.K. Rosing, Obesity and hyperlipidemia modulate alveolar bone loss in Wistar rats, J. Periodontol. 87 (2) (2016) e9-17.

[16] G.G. Nascimento, F.R. Leite, L.G. Do, K.G. Peres, M.B. Correa, F.F. Demarco, M.A. Peres, Is weight gain associated with the incidence of periodontitis? A systematic review and meta-analysis, J. Clin. Periodontol. 42 (6) (2015) 495-505.

[17] V.C. Colli, R. Okamoto, P.M. Spritzer, R.C. Dornelles, Oxytocin promotes bone formation during the alveolar healing process in old acyclic female rats, Arch. Oral Biol. 57 (9) (2012) 1290-1297.

[18] L.L. Bernardis, B.D. Patterson, Correlation between 'Lee index' and carcass fat content in weanling and adult female rats with hypothalamic lesions, J. Endocrinol. 40 (4) (1968) 527-528.

[19] T. Okamoto, M.C. de Russo, Wound healing following tooth extraction. Histochemical study in rats, Rev. Fac. Odontol. Aracatuba 2 (2) (1973) 153-169.

[20] T. Iizuka, S.C. Miller, S.C. Marks Jr., Alveolar bone remodeling after tooth extraction in normal and osteopetrotic (ia) rats, J. Oral Pathol. Med. 21 (4) (1992) $150-155$.

[21] J. Merzel, C.R. Salmon, Growth and the modeling/remodeling of the alveolar bone of the rat incisor, Anat. Rec. (Hoboken) 291 (7) (2008) 827-834.

[22] T.M. Manfrin, W.R. Poi, R. Okamoto, S.R. Panzarini, C.K. Sonoda, C.T. Saito, E.F. Hamanaka, C.M. Martins, Expression of OPG, RANK, and RANKL proteins in tooth repair processes after immediate and delayed tooth, J. Craniofac. Surg. 24 (1) (2013) e74-e80.

[23] A.L. Evans, M.A. Paggiosi, R. Eastell, J.S. Walsh, Bone density, microstructure and strength in obese and normal weight men and women in younger and older adulthood, J. Bone Miner. Res. 30 (5) (2015) 920-928.

[24] M.Y. Chan, S.A. Frost, J.R. Center, J.A. Eisman, T.V. Nguyen, Relationship between body mass index and fracture risk is mediated by bone mineral density, J. Bone Miner. Res. 29 (11) (2014) 2327-2335.

[25] R. Zura, M.J. Braid-Forbes, K. Jeray, S. Mehta, T.A. Einhorn, J.T. Watson, G.J. Della Rocca, K. Forbes, R.G. Steen, Bone fracture nonunion rate decreases with increasing age: a prospective inception cohort study, Bone 95 (2017) 26-32.

[26] M.R. Laurent, M.J. Cook, E. Gielen, K.A. Ward, L. Antonio, J.E. Adams,
B. Decallonne, G. Bartfai, F.F. Casanueva, G. Forti, A. Giwercman, I.T. Huhtaniemi, K. Kula, M.E.J. Lean, D.M. Lee, N. Pendleton, M. Punab, F. Claessens, F.C.W. Wu, D. Vanderschueren, S.R. Pye, T.W. O'Neill, E. Group, Lower bone turnover and relative bone deficits in men with metabolic syndrome: a matter of insulin sensitivity? The European Male Ageing Study, Osteoporos. Int. 27 (11) (2016) 3227-3237.

[27] C.J. Rosen, M.L. Bouxsein, Mechanisms of disease: is osteoporosis the obesity of bone? Nat. Clin. Pract. Rheumatol. 2 (1) (2006) 35-43.

[28] J.G. Neels, J.M. Olefsky, Inflamed fat: what starts the fire? J. Clin. Invest. 116 (1) (2006) 33-35.

[29] H. Tilg, A.R. Moschen, Adipocytokines: mediators linking adipose tissue, inflammation and immunity, Nat. Rev. Immunol. 6 (10) (2006) 772-783.

[30] G.V. Halade, M.M. Rahman, P.J. Williams, G. Fernandes, High fat diet-induced animal model of age-associated obesity and osteoporosis, J. Nutr. Biochem. 21 (12) (2010) 1162-1169.

[31] H. Goto, M. Osaki, T. Fukushima, K. Sakamoto, A. Hozumi, H. Baba, H. Shindo, Human bone marrow adipocytes support dexamethasone-induced osteoclast differentiation and function through RANKL expression, Biomed. Res. 32 (1) (2011) $37-44$.

[32] F. Xu, Y. Du, S. Hang, A. Chen, F. Guo, T. Xu, Adipocytes regulate the bone marrow microenvironment in a mouse model of obesity, Mol. Med. Rep. 8 (3) (2013) 823-828.

[33] J.M. Halleen, S. Raisanen, J.J. Salo, S.V. Reddy, G.D. Roodman, T.A. Hentunen, P.P. Lehenkari, H. Kaija, P. Vihko, H.K. Vaananen, Intracellular fragmentation of bone resorption products by reactive oxygen species generated by osteoclastic tartrate-resistant acid phosphatase, J. Biol. Chem. 274 (33) (1999) 22907-22910.

[34] J. Vaaraniemi, J.M. Halleen, K. Kaarlonen, H. Ylipahkala, S.L. Alatalo, G. Andersson, H. Kaija, P. Vihko, H.K. Vaananen, Intracellular machinery for matrix degradation in bone-resorbing osteoclasts, J. Bone Miner. Res. 19 (9) (2004) 1432-1440.

[35] G. Andersson, B. Ek-Rylander, K. Hollberg, J. Ljusberg-Sjolander, P. Lang, M. Norgard, Y. Wang, S.J. Zhang, TRACP as an osteopontin phosphatase, J. Bone Miner. Res. 18 (10) (2003) 1912-1915.

[36] P.V. Hauschka, J.B. Lian, D.E. Cole, C.M. Gundberg, Osteocalcin and matrix Gla protein: vitamin K-dependent proteins in bone, Physiol. Rev. 69 (3) (1989) 990-1047.

[37] N.K. Lee, H. Sowa, E. Hinoi, M. Ferron, J.D. Ahn, C. Confavreux, R. Dacquin, P.J. Mee, M.D. McKee, D.Y. Jung, Z. Zhang, J.K. Kim, F. Mauvais-Jarvis, P. Ducy, G. Karsenty, Endocrine regulation of energy metabolism by the skeleton, Cell 130 (3) (2007) 456-469.

[38] J. Street, M. Bao, L. deGuzman, S. Bunting, F.V. Peale Jr., N. Ferrara, H. Steinmetz, J. Hoeffel, J.L. Cleland, A. Daugherty, N. van Bruggen, H.P. Redmond, R.A. Carano, E.H. Filvaroff, Vascular endothelial growth factor stimulates bone repair by promoting angiogenesis and bone turnover, Proc. Natl. Acad. Sci. U. S. A. 99 (15) (2002) 9656-9661. 